\title{
Medical management in locally advanced and metastatic prostate cancer: Does changes in treatment policy have any specific effect on PSA levels?
}

\author{
Murat Bagcioglu ${ }^{1}$, Cristian Surcel ${ }^{2}$, Serkan Ozcan ${ }^{3}$, Cristian Mirvald ${ }^{2}$, Mehmet Ali Karagoz ${ }^{4}$, \\ Mert Ali Karadag ${ }^{1}$, Emre Huri ${ }^{5}$, Kemal Sarica ${ }^{6}$ \\ ${ }^{1}$ Urology Department Kafkas University Faculty of Medicine, Kars, Turkey; \\ ${ }^{2}$ Fundeni Clinical Institute, Center of Urologic Surgery and Renal Transplantation, Bucharest, Romania; \\ ${ }^{3}$ Urology Department Artvin State Hospital, Artvin, Turkey; \\ ${ }^{4}$ Urology Department Ankara Research and Training Hospital, Ankara, Turkey; \\ ${ }^{5}$ Urology Department Hacettepe University, Faculty of Medicine, Ankara, Turkey; \\ ${ }^{6}$ Urology Department Kartal Research and Training Hospital, Istambul, Turkey.
}

\section{Summary Objective: Androgen deprivation therapy (ADT) is commonly used as a first-line} treatment for locally advanced and metastatic prostatic cancer (Pca). There is no consensus about which alternative treatment should be used after the failure of initial ADT. We aimed to investigate the effect of changes in treatment on PSA and testosterone levels.

Material and methods: A total of 120 patients with an established diagnosis of either locally advanced or metastatic Pca in two different centers. Depending on the type of medical and/or surgical management protocol planned at initial presentation, all cases were divided into three main groups as follows. Group 1 ( $n$ : 80) included the patients who underwent medical management during whole follow-up period in whom the initial management protocol was later on switched to another medical treatment with different agents, Group 2 (n: 20) included patients who were initially treated with a medical management protocol and switched to surgical castration during follow-up evaluation and lastly Group 3 (n: 20) included the patients undergoing treated surgical castration as initial treatment modality without any further medical management protocol.

Results: Evaluation of our data did clearly demonstrate a statistically significant difference between the initial and final PSA as well as testosterone levels in Group 1 cases. Mean PSA and testosterone levels increased significantly in these cases despite a change in hormonal therapy by using another agent for androgen deprivation. Cases in Group 2 and 3 cases did not show any statistically significant difference with respect to the mean PSA as well as testosterone values during the same follow-up period.

Conclusions: Our data clearly indicated that in case of a biochemical progression, switching into another alternative medical treatment was not effective enough in limiting the rising PSA levels in a statistically significant manner when compared with the approaches of switching to surgical castration after initial medical treatment or continuing with regular and close follow-up after initial surgical castration alone.

KEY WORDS: Prostats cancer; PSA; Hormonal therapy; Treatment changes.

Submitted 21 October 2017; Accepted 20 November 2017

\section{INTRODUCTION}

With a 1.1 million new cases being estimated in 2012, prostate cancer $(\mathrm{Pca})$ is the second most frequently diagnosed cancer in males and it represents the fifth leading cause of cancer deaths worldwide (1). Although surgical and/or medical management principles are well established in all the stages of the disease, treatment options for cases with a life expectancy of less than 10 years remain still controversial.

Related with this issue, in addition to its successful outcomes as the first-line treatment option in patients with both locally advanced and metastatic Pca, androgen deprivation therapy (ADT) has also proven to be successful as the second most common treatment after surgery for localized Pca $(2,3)$. However, there is an ongoing controversy regarding the optimal use of ADT in these cases where medical [combined androgen blockade (CAB), gonadotropin-releasing hormone (GnRH) agonists alone, GnRH antagonists] or surgical (bilateral orchiectomy) options are being applied with different success as well as undesired effect rates.

Among the above mentioned options, GnRH agonists are generally preferred as ADT due to their reduced psychological morbidity and almost equivalent efficacy to surgical castration (2) but these agents have some adverse effects including testosterone surges, bone loss, and metabolic complications. Moreover, biochemical progression which may end up with an advanced stage has also been reported in the majority of cases undergoing medical therapy with GnRH agonists. Thus, it seems clear that initial ADT may result in an unsuccessful outcome and again there is no established approach in such cases with ADT failure. Last but not least, there is also no consensus regarding the type of the second-line treatment (another GnRH agonist or GnRH antagonist, antiandrogens, estrogens, steroids, or orchiectomy) to be used in these cases.

In this study we aimed to investigate the effect of changes in medical treatment on prostate specific antigen (PSA) and testosterone levels. 


\section{MATERIALS AND Methods}

A total of 120 patients with an established diagnosis of either locally advanced or metastatic Pca in two different centers between 2003 and 2014 were included into the study program. The study protocol was approved by the local ethics committee (number 2680576354-050-99/97), and a written informed consent stating all details of the investigation protocol was obtained from all of patients before including data from the hospital registry files.

In addition to the clinical stage of PCa according to TNM classification via digital rectal examination, other relevant data were obtained by findings of computed tomography, transrectal ultrasonography, bone X-ray and bone imaging using 99mTc-methylene-diphosphonate from patient registry files. Regarding the inclusion criteria for the study protocol, patients with an initial diagnosis of locally advanced prostate cancer who are not planned for radical prostatectomy or patients with metastatic Pca were included into the study program. Within the framework of inclusion criteria, in addition to the cases undergoing medical androgen deprivation by applying initial treatment protocols with leuprolide acetate $11.25 \mathrm{mg}$ (Lucrin Depot, Abbott Laboratories, Saint Laurent, 2C, Canada), goserelin $10.8 \mathrm{mg}$ (Zoladex, AstraZeneca Canada, Missisauga, ON, Canada) or leuprolide acetate $22.5 \mathrm{mg}$ (Eligard, Astellas Pharma Inc., Tokyo, Japan) and bicalutamide $50 \mathrm{mg}$ (Casodex, AstraZeneca Canada) as preferred antiandrogen agents, data were also obtained from the cases undergoing surgical castration. All cases had an adequate long-term follow-up data for a reliable interpretation. On the other hand, exclusion criteria included the patients with organ confined prostate cancer without any local extension and/or metastasis and that undergoing simultaneous radiation therapy. All data derived from the patient registry files were carefully searched and recorded for the clinical variables including age at diagnosis and initial treatment of prostate cancer, Gleason score values, presence or absence of bone metastasis (single, multiple or no metastasis), timing and other characteristics of medical management with GnRH agonists (primary treatment and secondary treatment) and disease status (assessment of biochemical recurrence, serum PSA values, testosterone levels). Biochemical recurrence was defined as a PSA value of at least $2 \mathrm{ng} / \mathrm{ml}$ followed by another two consecutive rises in serum PSA. Depending on the type of medical and/or surgical management protocol planned at initial presentation, all cases were divided into three main groups as follows. Group 1 (n: 80) included the patients who underwent medical management during whole follow-up period in whom the initial management protocol was later on switched to another medical treatment protocol with different agents; Group 2 (n: 20) included patients who were initially treated with a medical management protocol and switched to surgical castration during follow-up; Group 3 (n: 20) included the patients undergoing surgical castration as the initial treatment modality without any further change of medical management protocol. During the follow-up period, all patients were seen and evaluated regularly at every 3 months. Patients in all groups were comparative- ly evaluated with respect to the age, bone metastasis and Gleason score values. The results are presented as mean \pm standard deviation (SD). Statistical significance analysis was obtained by using the Statistical Package for the Social Science (SPSS, Inc., Chicago, Illinois, USA) version 15.0. Mann-Whitney U test and Wilcoxon tests were used for the statistical analyses. A p value of less than 0.05 was considered to be statistically significant.

\section{RESULTS}

Of all the 120 patients included and evaluated, the overall mean age value at the time of initial diagnosis was $67.0 \pm$ 1.5 years, and the mean follow-up duration was $51.3 \pm 3.1$ months (Table 1). The overall mean prostate gland volume prior to treatment was $40.21 \pm 1.82 \mathrm{ml}$. While the mean PSA value at the time of diagnosis was $136.88 \pm 9.66$ $\mathrm{ng} / \mathrm{ml}$; Gleason 7 and ECOG 1 were the most frequent scores in our study group. Out of 120 patients 24 had locally advanced Pca, 36 had bone metastasis, 32 had lymph node metastasis, 16 had visceral metastasis and lastly 12 were with unknown extension (Table 2).

As stated above, in Group 1 medical management was initiated with a certain protocol in 80 patients (66.6\%) and the medical management protocol was switched to another medical protocol with different agents after pro-

Table 1.

Patients' demographics.

\begin{tabular}{lcccc} 
& Group 1 & Group 2 & Group 3 & P value \\
\hline Prostate volume & $39.31 \pm 2.11$ & $41.14 \pm 5.48$ & $43.04 \pm 5.38$ & 0.831 \\
\hline Age (at biopsy) & $67.48 \pm 1.95$ & $62.80 \pm 2.57$ & $69.20 \pm 4.22$ & 0.587 \\
\hline PSA level (at biopsy) & $135.61 \pm 12.16$ & $154.22 \pm 30.07$ & $124.89 \pm 14.63$ & 0.683 \\
\hline PSA: Prostate specific antigen. & & &
\end{tabular}

Table 2.

Clinical stage of Pca, Hormonal therapy first and second treatment.

\begin{tabular}{|lcc|}
\hline Clinical stage & $\mathbf{N}^{\mathbf{0}}$ & Percent \\
\hline Locally advanced, M0 & 24 & 20.0 \\
\hline M+, bone disease & 36 & 30.0 \\
\hline M+, lymph node disease & 32 & 26.7 \\
\hline M+, visceral disease & 16 & 13.3 \\
\hline M+, unknown extension & 12 & 10.0 \\
\hline Total & 120 & 100.0 \\
\hline Hormonal therapy first treatment & & \\
\hline Leuprolide & 20 & 16.7 \\
\hline Goserelin & 48 & 40.0 \\
\hline Triprorelin & 32 & 26.7 \\
\hline Surgical castration & 20 & 16.7 \\
\hline Total & 120 & 100.0 \\
\hline Hormonal therapy second treatment & & \\
\hline Leuprolide & 28 & 23.3 \\
\hline Goserelin & 20 & 16.7 \\
\hline Triprorelin & 20 & 16.7 \\
\hline Histrelin & 12 \\
\hline Surgical castration & 40 & 10.0 \\
\hline Total & 120 \\
\hline ADT: Androgen Deprivation Therapy. & 100.0 \\
\hline
\end{tabular}


Table 3.

Antiandrogen treatment and adjuvant treatment in first and second period of treatment.

\begin{tabular}{|lcc|}
\hline $\begin{array}{l}\text { Antiandrogen in first treatment period } \\
\text { Flare-up prevention }\end{array}$ & $\mathbf{N}^{\circ}$ & Percent \\
\hline Max. ADT & 76 & 63.3 \\
\hline Total & 120 & 36.7 \\
\hline Antiandrogen in second treatment period & 100.0 \\
\hline No & 32 & 26.7 \\
\hline Yes & 72 & 60.0 \\
\hline Surgical castration & 36 & 13.3 \\
\hline Total & 120 & 100.0 \\
\hline Adjuvant treatment in first treatment period & \\
\hline None & 84 & 70.0 \\
\hline Zolendronic acid & 28 & 23.3 \\
\hline Denosumab & 8 & 6.7 \\
\hline Total & 120 & 100.0 \\
\hline Adjuvant treatment in second treatment period & \\
\hline None & 64 & 53.3 \\
\hline Switched to Denosumab & 44 \\
\hline Zolendronic acid & 12 & 10.0 \\
\hline Total & 30 & 100.0 \\
\hline ADT: Androgen Deprivation Therapy. & \\
\hline
\end{tabular}

Table 4.

Timing of treatments.

\begin{tabular}{|lcccccccc|}
\hline & $\begin{array}{c}\text { From biopsy } \\
\text { to first PSA on } \\
\text { treatment (month) }\end{array}$ & $\begin{array}{c}\text { From first PSA } \\
\text { to switch of } \\
\text { treatment (month) }\end{array}$ & \multicolumn{2}{c|}{$\begin{array}{c}\text { From switch of } \\
\text { treatment to } \\
\text { last PSA (month) }\end{array}$} & PSA at switch \\
\hline \multicolumn{2}{c}{ Mean } & \multicolumn{2}{c}{ Mean } & \multicolumn{2}{c|}{ Mean } & \multicolumn{2}{c|}{ Mean } \\
\hline Statistic & Std. error & Statistic & Std. error & Statistic & Std. error & Statistic & Std. error \\
\hline Group 1 & 8,20 & .691 & 22.40 & 1.963 & 26.65 & 3.761 & 7.6600 & .75248 \\
\hline Group 2 & 11,00 & 2.280 & 25.00 & 4.301 & 24.60 & 5.501 & 7.8060 & 1.50512 \\
\hline Group 3 & 7.60 & .245 & & & 60.40 & 5.591 & & \\
\hline
\end{tabular}

Table 5.

First-last PSA levels and first-last testosterone levels for groups.

\begin{tabular}{|lcccccc|}
\hline $\begin{array}{c}\text { First PSA level } \\
\text { on treatment }\end{array}$ & $\begin{array}{c}\text { Last PSA } \\
\text { level }\end{array}$ & P value & $\begin{array}{c}\text { First testosterone } \\
\text { level on treatment }\end{array}$ & $\begin{array}{c}\text { Last testosterone } \\
\text { level }\end{array}$ & P value \\
\hline Group 1 & $5.16 \pm 0.59$ & $9.20 \pm 0.97$ & 0.000 & $27.08 \pm 1.45$ & $38.50 \pm 1.66$ & 0.000 \\
\hline Group 2 & $7.36 \pm 1.00$ & $7.56 \pm 1.33$ & 0.686 & $30.30 \pm 4.4 \mathrm{X}$ & $41.5 \mathrm{X} \pm 3.90$ & 0.223 \\
\hline Group 3 & $6.77 \pm 1.45$ & $9.01 \pm 1.37$ & 0.138 & $24.90 \pm 1.88$ & $31.30 \pm 5.31$ & 0.225 \\
\hline PSA: Prostate specific antigen. & & & & \\
\hline
\end{tabular}

Figure 1.

Timing of treatments.

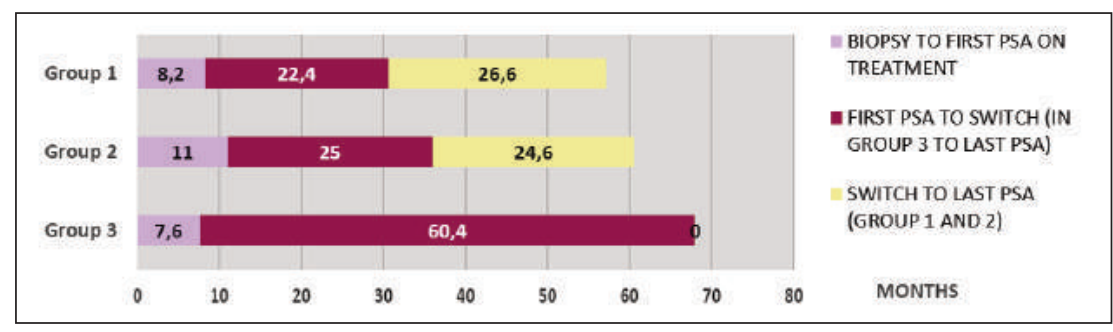

progression during follow-up. Last, 20 patients (16.6\%) in Group 3 were treated with surgical castration and followed up regularly without any further change of treatment protocol.

There was no statistically significant difference between the groups with respect to the mean age, mean initial PSA levels and Gleason score values as well as the mean prostate volume values ( $p>0.05$ ).

Regarding the agents used within the framework of medical management plan in Groups 1 and 2, 20 patients received leuprolide acetate as the initial medical agent for androgen deprivation; 48 patients with goserelin and 32 patients with triptorelin (Table 2). As stated above, the initial hormonal management plan was changed and continued with another medical agent after assessing progression of the disease status: 28 patients were treated with leuprolide, 20 patients with goserelin, and 20 patients with triptorelin and other 12 patients with histrelin. On the other hand as shown in Table 2, a total of 40 patients were treated with surgical castration in Group 2, as secondary treatment after assessment of progression, and in Group 3, as initial treatment. Maximum androgen blockage was applied in 44 patients and antiandrogens were administered only to prevent flare-up phenomenon in other 76 patients (Table 3 ). No adjuvant treatment was administered in 84 patients, whereas zoledronic acid was administered in 28 cases due to bone problems and denosumab was added to the initial treatment in another 8 cases. Of all these cases, although 12 patients continued their management with zoledronic acid, management was switched to denasumab in 44 cases (Table 3). Time intervals of the periods of treatment are shown in Table 4 and Figure 1.

Evaluation of our data did clearly demonstrate a statistically significant difference between the initial and final PSA as well as testosterone levels in Group 1 cases (Table 5). Mean PSA and testosterone levels increased significantly in these cases despite a change in hormonal therapy by using another agent for androgen deprivation. In other words, switching to another medical management protocol with another agent did not affect the rising PSA level in these cases.

In Group 2 and 3 cases mean PSA and testosterone values did not show any statistically significant difference during the same follow-up period. gression. In Group 2, 20 patients (16.6\%) were initially treated with a medical treatment protocol but the management plan was switched to surgical castration after

\section{Discussion}

An estimated 242000 men were diagnosed with pCa in 2012 only in the United States, and probably 28000 of 
these men will die from the disease $(4,5)$. For over two decades, bilateral orchiectomy and GnRH analogues have been accepted as equivalent therapeutic options (6). GnRH are largely preferred by urologists and patients for the treatment of advanced and metastatic $\mathrm{PCa}$, although they have similar efficacy to a bilateral orchiectomy. Although an initial response to medical castration is often reported, resistance to GnRH agonists in some patients with Pca has been previously reported $(7,8)$. After failure of the initial hormonal therapy, the second line therapy has not yet been completely established and there is no consensus on which alternative treatment alternative should be used (e.g., switching to another GnRH agonist or GnRH antagonist or using antiandrogens, estrogens, steroids, or surgical castration).

Patients with carcinoma of the prostate eventually progress despite castration. Continuing maximal testicular androgen suppression is debatable in this situation (9). These data have been argued by 2 studies that established only a survival advantage in patients continuing GnRH analogues during second- and third-line therapies in case of failure $(10,11)$.

The modest potential benefits of a continuing castration outweigh the minimal risk of treatment despite lack of prospective data in the literature. In addition, all treatment options have been conducted in men under androgen-suppression therapy and such therapy should be continued indefinitely in these patients. Even though we did not determine the survival benefits in second-line therapy, we noted ongoing PSA progression in all three groups.

This progression was statistically significant in patients who had been previously treated with medical treatment and switched to another medical alternative. Unlike surgically castrated patients, it seemed that switching to another medical castration regime increased the speed of the biochemical progression process.

This ongoing biochemical progression in all three groups may be explained by the aggressive tumor biology of the cases. In fact, as noted previously, the initial PSAs (> 100 $\mathrm{ng} / \mathrm{ml}$ ) and Gleason scores of our patients were very high.

Taking all these facts into account, in this present study including a large group of cases from two different centers we aimed to evaluate the possible effects of changes in treatment policies during follow-up on the prostatespecific antigen (PSA) in cases with locally advanced prostate cancer.

Evaluation of our results clearly revealed that biochemical progression may occur despite a change in treatment policy by switching to another medical treatment modality even in patients undergoing maximal androgen blockade as initial treatment protocol. Although a change in the medical therapy protocol by using another antiandrogen agent was not found to prevent the progression and rise in both PSA as well as testosterone values in these cases, possible disease progression seemed to be stabilized in patients in Group 2 and Group 3 who underwent surgical castration initially after failure of initial medical management or directly as initial approach. In other words, we were able to show that after biochemical progression, switching to surgical castration after a certain period of medical treatment or continuing with surgical castration alone stabilized PSA progression in comparison with medical castration plus another medical castration regimen.

On the other hand, when we evaluated testosterone levels in cases of all study groups, these values were found to be at castrate levels despite rising PSA values. The biochemical progression states was found to be independent of androgen receptors in the three groups.

Regarding the management of metastatic prostate carcinoma after the failure of initial hormonal therapy, the Guidelines of European Association of Urology (EAU) have recommended that these patients should not be started a second-line therapy unless their testosterone serum levels are $<50 \mathrm{ng} / \mathrm{dL}$ (Level of evidence: $4 \mathrm{~A}$, Grade of recommendation: A) and PSA serum levels are $>2 \mathrm{ng} / \mathrm{mL}$ to ensure correct interpretation of therapeutic efficacy (Level of evidence: 4 B, Grade of recommendation: B). We used both recommendations in our patients when switching to another treatment alternative (2). The EAU panel on this issue also claimed that there is no evidence for the treatment of non-metastatic castration resistant prostate cancer outside of a clinical trial (Level of evidence: 3, Grade of recommendation: A) and that men who are under maximal androgen blockade should stop antiandrogen therapy once progression of PSA is occurred (Level of evidence: 2a, Grade of recommendation: A). There is not any clear-cut recommendation for the use of the most effective drug as the second line treatment (i.e., chemotherapy or hormone therapy) since no reliable predictive factors exist (Level of evidence: 3, Grade of recommendation A).

Our choice in continuing with another maximal androgen blockade protocol in Group 1 patients without stopping the antiandrogen therapy was based on the lack of clear evidence about this topic in the literature despite a Grade A recommendation for cessation of antiandrogen therapy.

As stated above, we used sole PSA progression in our study in order to evaluate the response of the patients to the given treatment alternatives. Even though there is no consensus about the duration as well as the degree of a decline in PSA level, many research studies have used PSA as a marker of response like in our study. Although PSA is a rapid screening tool to evaluate the effect of newer molecules, there is an ongoing and conflicting debate about the role of PSA as a surrogate marker in initial treatment failures of prostate carcinoma. TRICOM and sipuleucel-T studies have revealed a statistically significant overall survival without any PSA change and raise questions about the value of PSA response for noncytotoxic, non-hormonal drugs (12-14).

Our current study may have some limitations, in particular not including the PSA velocity and doubling time and the cancer-specific and disease-specific survival rates of the patients. Furthermore, we failed to conduct a cost effectiveness and quality of life analysis in and among the 3 groups. On the other hand, the retrospective nature of our investigation was also another limitation. There should have been be strict follow-up criteria for evaluating these parameters, but we were unable to perform it due to low sociocultural level of the patients in both 
Turkey and Romania. In our opinion, if the study was designed as prospective randomized study the power of our investigation would have been much improved. On the other hand this type of study are lacking in the literature about second-line treatments after initial failure of the first treatment of PCa.

However despite the above mentioned limitations we believe that our study with a reasonably longer follow-up period of approximately 5 years in a relatively large group of cases from two centers of different countries (i.e., Turkey and Romania) will be contributive enough to the existing data in about this topic.

\section{Conclusions}

In the light of our findings and of the so far reported data in the literature, there is an ongoing controversy concerning the assessment and application of the secondline treatment modalities in patients with metastatic and non-metastatic, castration-resistant prostate carcinoma. Our data clearly indicated that in case of a biochemical progression, switching into another medical treatment alternative was not effective enough in limiting the rising PSA levels in a statistically significant manner when compared with the approaches of switching to surgical castration after initial medical treatment or continuing with regular and close follow-up after an initial surgical castration alone. However we believe that further prospective randomized studies with larger group of cases and additional predictive parameters are certainly required in this specific group of cases to outline a true and effective algorithm.

\section{REFERENCES}

1. Torre LA, Bray F, Siegel RL, et al. Global cancer statistics, 2012. CA Cancer J Clin. 2015; 65:87-108.

2. Mottet N, Bellmunt J, Patient EB, et al. EAU 2015 Guidelines. Guidelines on Prostate Cancer.
3. Cooperberg MR, Grossfeld GD, Lubeck DP, Carroll PR. National practice patterns and time trends in androgen ablation for localized prostate cancer. J Natl Cancer Inst. 2003; 95:981-989.

4. Siegel R, Naishadham D, Jemal A. Cancer statistics, 2012. CA Cancer J Clin. 2012; 62:10-29.

5. Sutcliffe S, Colditz GA. Prostate cancer: is it time to expand the research focus to early-life exposures? Nat Rev Cancer. 2013;13:208518.

6. Zoladex Prostate Study Group.Goserelin versus orchiectomy in the treatment of advanced prostate cancer: final results of a randomized trial. Urology. 1995; 46:220-226.

7. Eisenberger MA, Blumenstein BA, Crawford ED, et al. Bilateral orchiectomy with or without flutamide for metastatic prostate cancer. N Engl J Med. 1998; 1339:1036-1042.

8. Curry EA, Sweeney CJ. Resistance to luteinizing hormone releasing hormone agonist therapy for metastatic prostate cancer. J Urol. 2002; 168:193.

9. Manni A, Bartholomew M, Caplan R, et al. Androgen priming and chemotherapy in advanced prostate cancer: evaluation of determinants of clinical outcome. J Clin Oncol. 1998; 6:1456-1466.

10. Hussain M, Wolf M, Marshall E, et al. Effects of continued androgen-deprivation therapy and other prognostic factors on response and survival in phase II chemotherapy trials for hormonerefractory prostate cancer: a Southwest Oncology Group report. J Clin Oncol. 1994; 12:1868-1875.

11. Taylor CD, Elson P, Trump DL. Importance of continued testicular suppression in hormone-refractory prostate cancer. J Clin Oncol. 1993; 11:2167-2172.

12. Kantoff PW, Schuetz TJ, Blumenstein BAet al. Overall survival analysis of a phase II randomized controlled trial of a Poxviralbased PSA-targeted immunotherapy in metastatic castration-resistant prostate cancer. J Clin Oncol. 2010; 28:1099-1105.

13. Small EJ, Schellhammer PF, Higano CS, et al. Placebo-controlled phase III trial of immunologic therapy with sipuleucel-T (APC8015) in patients with metastatic, asymptomatic hormone refractory prostate cancer. J Clin Oncol. 2006; 24:3089-3094.

14. Bellmunt J, Rosenberg JE, Choueiri TK. Recent progress and pitfalls in testing novel agents in castration-resistant prostate cancer.

\section{Correspondence}

Murat Bagcioglu, MD

dr.muratbagcioglu@hotmail.com

Mert Ali Karadag, MD

Urology Department Kafkas University Faculty of Medicine, Kars, Turkey

Cristian Surcel, MD

drsurcel@gmail.com

Cristian Mirvald, MD

Fundeni Clinical Institute, Center of Urologic Surgery and Renal

Transplantation Country, Bucharest, Romania

Serkan Ozcan, MD

Urology Department Artvin State Hospital, Artvin, Turkey

Mehmet Ali Karagoz, MD

Urology Department Ankara Research and Training Hospital, Ankara, Turkey

Emre Huri, MD

emrehuri@gmail.com

Urology Department Hacettepe University, Faculty of Medicine, Ankara, Turkey

Kemal Sarica, MD

Urology Department Kartal Research and Training Hospital, Istambul, Turkey 